2017-9

\title{
A Whooping Cough Education Module for WIC Clients in Utah
}

Karlen E. Luthy

Brigham Young University - Provo

Alicia Anderson

Brigham Young University - Provo

Janelle L. B. Macintosh

Brigham Young University - Provo, janelle-macintosh@byu.edu

Renea L. Beckstrand

Brigham Young University - Provo

Lacey M. Eden

Brigham Young University - Provo

Follow this and additional works at: https://scholarsarchive.byu.edu/facpub

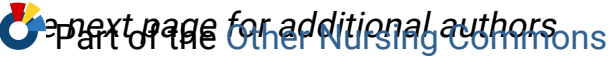

\section{Original Publication Citation}

Luthy, K. E., Anderson, A.**, Macintosh, J. L. B., Beckstrand, R. L., Eden, L. M., Amy, R., \& Macintosh, C. I. (2017). A whooping cough education module for WIC clients in Utah. MCN: The American Journal of Maternal/Child Nursing, 42(5), 283-288.

\section{BYU ScholarsArchive Citation}

Luthy, Karlen E.; Anderson, Alicia; Macintosh, Janelle L. B.; Beckstrand, Renea L.; Eden, Lacey M.; Amy, Ryan; and Macintosh, Christopher I., "A Whooping Cough Education Module for WIC Clients in Utah" (2017). Faculty Publications. 5205.

https://scholarsarchive.byu.edu/facpub/5205

This Peer-Reviewed Article is brought to you for free and open access by BYU ScholarsArchive. It has been accepted for inclusion in Faculty Publications by an authorized administrator of BYU ScholarsArchive. For more information, please contact ellen_amatangelo@byu.edu. 


\section{Authors}

Karlen E. Luthy, Alicia Anderson, Janelle L. B. Macintosh, Renea L. Beckstrand, Lacey M. Eden, Ryan Amy, and Christopher I. Macintosh 

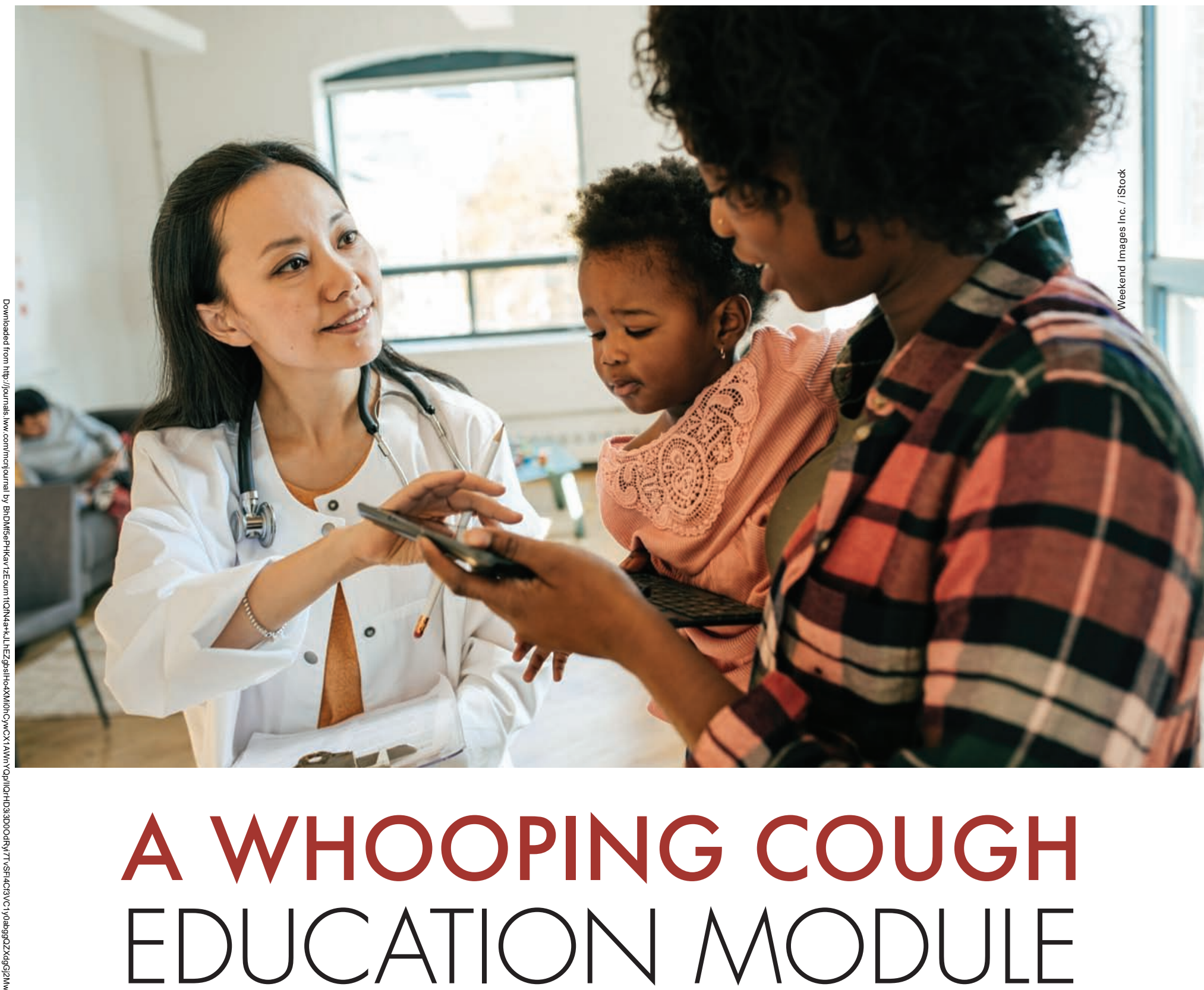

for WIC Clients in Utah

\section{Abstract}

Background: Clients in the Special Supplemental Nutrition Program for Women, Infants, and Children (WIC) are required to complete education modules quarterly to maintain eligibility. The purposes of this project were to: (1) create a whooping cough vaccination education module for WIC clients; (2) evaluate baseline perceptions of WIC clients on the whooping cough vaccine and disease; and (3) evaluate whooping cough knowledge following completion of the module. Problem: A decline in vaccination rates among infants and children using WIC services was reported by a local WIC program director who requested whooping cough vaccination education materials. This quality improvement project included development of a whooping cough
Karlen E. Luthy, DNP, FNP, Alicia Anderson, MS, RN, Janelle Macintosh, PhD, RN, Renea L. Beckstrand, PhD, RN, Lacey M. Eden, MS, FNP, Ryan Amy, MS IT, and Christopher I. Macintosh, PhD, RN 
education module and evaluation of learning. Methods: Learning was evaluated using a pre- and posttest design. Client feedback was solicited via open-ended questions. Quantitative analysis was performed on visual analog-type questions with paired $t$-tests and a Cohen's $d$. Content analysis was conducted on open-ended items. Interventions: The module was designed by a team of vaccination experts and included general definitions, signs and symptoms during the three stages of disease, recommendations to prevent whooping cough, and vaccination recommendations. Learning of users of the module was then evaluated. Results: After using the module, clients indicated they were significantly more likely to vaccinate themselves and their child against whooping cough, and to recommend the vaccination to their family members. The greatest concern of participants about whooping cough was how it affected infants. Participants reported they learned new information on disease seriousness, recognition of symptoms, and treatment options but still requested additional information on the whooping cough disease and vaccine. Conclusions: A whooping cough education module is an effective strategy to improve whooping cough knowledge and promote the whooping cough vaccine.

Key words: Education; Nurse; Vaccination; Whooping cough; WIC.

$\mathrm{P}$ rtussis infection, also known as whooping cough, is a highly contagious disease caused by the Bordetella pertussis bacterium. Whooping cough is well known for causing uncontrollable and even violent coughing that makes it difficult to breathe (Centers for Disease Control and Prevention [CDC], 2016). Upon initial infection, whooping cough often presents like a cold with symptoms such as mild cough, watery eyes, runny nose, and fever. In adults, whooping cough can cause a 100-day cough (CDC, 2017a). However, whooping cough is much more dangerous in young children and can result in death, especially for babies less than 1-year old (CDC, 2017a).

The CDC (2014) recommends routine whooping cough vaccination with diphtheria, tetanus, and pertussis (DTaP) at 2 months, 4 months, 6 months, 15 to 18 months, and 4 to 6 years of age. At age 11 years, the child should be vaccinated again with a booster known as the Tdap. The CDC (2014) recommends a Tdap booster in the third trimester of every pregnancy and for family members and caregivers who will have contact with infants (CDC, 2017b).

Despite the notable benefits of vaccinations, some states, such as Utah, struggle with low vaccination rates (Luthy, Miner, Faux, \& Sperhac, 2010). Evidence of low vaccination rates has become apparent in the last 5 years as Utah has had several outbreaks of whooping cough and measles. In 2012, Utah experienced a whooping cough outbreak so severe it rivaled the 1946 outbreak that took place before the development of a vaccination (Leonard, 2012).

The Special Supplemental Nutrition Program for Women, Infants, and Children (WIC) was first established in 1972 (United States Department of Agriculture [USDA], 2013). In Utah, approximately 66,000 lowincome mothers, infants, and children receive assistance from Utah's WIC program (Higley, 2013). To maintain eligibility, WIC clients are required to complete quarterly health education modules/classes. Creating and delivering specific whooping cough education for WIC participants may help increase vaccination rates among WIC infants and children.

The Utah county in which this intervention took place is one of the largest WIC programs in Utah, providing services for approximately 12,000 women, of whom need options for WIC-approved health education. In 2014, the Utah State Health Department reported a marked decline in vaccination rates among infants and children who used WIC services, which mirrored vaccination rates among WIC infants and children nationwide. National vaccination rates among WIC infants and children were so concerning that the White House issued an Executive Memorandum in 2000 to focus resources and efforts on increasing immunization rates (USDA, 2016a). To improve whooping cough vaccination rates of Utah WIC clients, the WIC director requested whooping cough education materials for clients. Although there were no previous data specifically identifying a lack of education as the primary reason for decreasing vaccination rates, it was assumed that Utah WIC parents may not be aware of the frequency of outbreaks in Utah and may benefit from an education module.

Knowing that WIC clients typically change their health behaviors after completing approved WIC health education (Utah Department of Health, 2013), creating a whooping cough education module for WIC clients was an opportunity to promote whooping cough vaccination as part of a quality improvement (QI) project. Therefore, the purposes of this project were to: (1) create a whooping cough vaccination education module for WIC clients in a Utah county; (2) evaluate baseline perceptions of WIC clients about the whooping cough vaccine and disease; and (3) evaluate the effectiveness of the education module on improving WIC clients' knowledge of whooping cough vaccine and disease.

\section{Project Questions}

1. What is the greatest concern of clients enrolled in WIC in a large Utah county (participants) prior to education on whooping cough?

2. How likely are participants to indicate they plan to vaccinate themselves or their children against whooping cough before and after completing the whooping cough education module?

3. How likely are participants to indicate they plan to recommend the whooping cough vaccination to their family members before and after completing the whooping cough education module? 
4. What new knowledge will participants gain from completing the education module?

5. What questions about whooping cough will participants have after completing the education module?

\section{Methods}

Institutional Review Board approval was obtained prior to development of the QI project on whooping cough education for WIC clients. The whooping cough education module included a pre- and posttest questionnaire with open-ended and visual analog questions.

\section{Context}

The whooping cough education module was developed specifically for WIC clients, knowing that routine whooping cough vaccinations are recommended for women, infants, and children. The module was designed by a team of vaccination experts from Utah pediatric clinics, pharmaceutical companies, the local Utah health department, and Utah nurses, as well as Utah WIC employees. It included definitions of whooping cough, signs and symptoms of disease, recommendations to prevent whooping cough, and whooping cough vaccination recommendations. The text was evaluated by an expert for readability and literacy level, which was determined to be at a 7 th grade to 8 th grade reading level. Prior to implementation, the module was pretested with 20 women in a different county who were between 20 and 40 years old and who were mothers of WIC-aged infants and children.

\section{Intervention}

The education was delivered through WordPress that was hosted at the WIC education site openwic.com. A recruitment flyer was distributed in all four WIC offices within the county, inviting WIC clients to complete the online whooping cough education module. On three separate days, a member of the research team was onsite to assist WIC clients to access the education module on laptop computers and/or tablets. Prior to beginning the module, participants read an informed-consent document. Consent was implied if the WIC client opted to complete the whooping cough module. Participants were asked to rate how likely they were to vaccinate themselves, vaccinate their children, and request other family members to get vaccinated before completing the education module. A $\$ 20$ gift card was offered as an incentive.

Because both men and women are eligible to complete quarterly WIC education, both genders were eligible to complete the whooping cough education module as part of their quarterly education requirement. Participants were required to speak fluent English because the whooping cough education module was only available in English.

\section{Study of the Intervention}

To assess the impact of the intervention, a pre- and posttest questionnaire was used (Figures 1 and 2 Supplemental Digital Content, http://links.lww.com/MCN/A41). The pretest questionnaire included four questions: one open-ended question and three visual analog scales. The open-ended

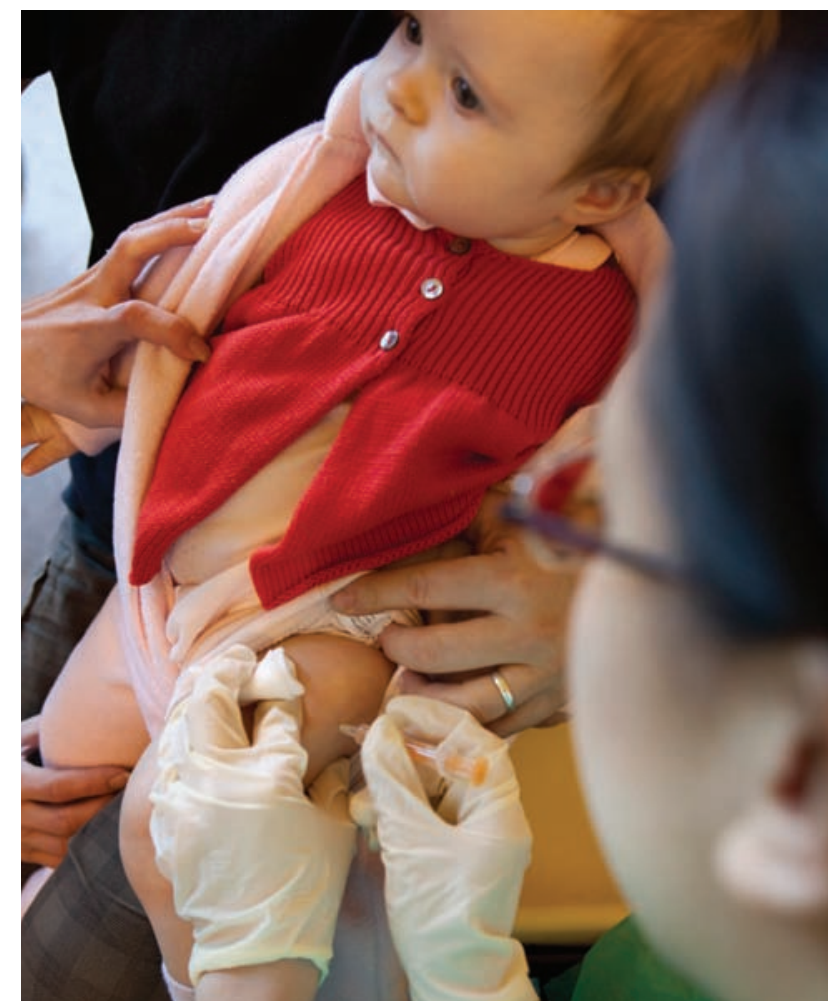

A whooping cough education module was created for the Special Supplemental Nutrition Program for Women, Infants, and Children in Utah.

question asked participants to share their greatest concern about whooping cough. For the visual analog scales, participants were asked to rate how likely they were to vaccinate (1) themselves, (2) their child, and (3) how likely they were to recommend whooping cough vaccination for their family. Participants rated their response on a scale of 0 to 100 by sliding the visual scale cursor to a point that best represented their perception $(0=$ not at all, $100=$ definitely $)$.

After the participant completed the module, they were asked the same three visual analog scale questions rated on the same scale of 0 to $100(0=$ not at all, $100=$ definitely). As part of the posttest questionnaire, participants were asked two additional open-ended questions: (1) what new piece of information they learned in the education module; and (2) what whooping cough questions the participant still had after completing the education module.

\section{Measures}

Prior to distribution, the questionnaires were pretested by 20 women in a different county who were between 20 and 40 years old and who were mothers of WIC-aged infants and children. The women provided feedback about the pretest and posttest questionnaires. Their suggestions included recommendations for minor changes in wording, which were made. 


\section{Analysis}

Quantitative analysis was performed using IBM SPSS Statistics, Armonk, NY version 23. Descriptive statistics were calculated for study variables. Comparisons between pretest and posttest responses were made using paired $t$-tests. Effect size for significant pairwise comparisons was calculated using Cohen's $d$. Responses to openended items were analyzed by two researchers, each of whom independently conducted a content analysis and identified preliminary codes. They then discussed preliminary codes until consensus was reached.

\section{Results}

A convenience sample of WIC clients $(n=104)$ in a large Utah county was recruited to participate in this project; 104 participants completed pretest questions and 101 participants completed posttest questions. Ninety-eight participants completed both the pre- and posttest questions (Table 1). Data on the number of participants who viewed the module but did not complete the pretest or the posttest were not collected. Because recruitment flyers were freely distributed to WIC clients who had the option of completing the whooping cough education module at home, the number of participants who declined to view the module is unknown. Demographic information about project participants was not collected.

\section{Analyses of Likelihood of Vaccination Questions}

Visual analog scores were higher on average at posttest when compared to pretest, indicating participants felt they were more likely to seek vaccination for themselves, their children, and other family members. Comparisons between pretest and posttest responses were conducted using paired $t$-tests and effect size for significant pairwise comparisons was calculated using Cohen's $d$. Results for paired $t$-tests are reported on Table 2. There were significant differences between pretest and posttest responses for all three visual analog questions. Participants reported they were more likely to seek whooping cough vaccinations for themselves, their children, and their family members after completing the module. Effect size for differences in probability of self-vaccination $(d=.63)$ indicated a medium effect, vaccinating children $(d=.33)$ indicated a small effect, and asking family members to be vaccinated $(d=.83)$ indicated a large effect.

\section{Greatest Concern}

All 104 participants answered the open-ended question on their greatest whooping cough concern during the pretest. Content analysis revealed three common main themes: (1) concerns about whooping cough and infants $(n=33)$; (2) worry about whooping cough mortality $(n=22)$; and (3) lack of knowledge $(n=18)$.

The most common theme demonstrated general community knowledge about the specific danger of whooping cough in the infant population.

I have a 3-month old baby, so I worry about her catching whooping cough since she has only received her first set of vaccines and isn't fully immunized against it. I know that it can cause serious illness and even death in infants.

Another participant shared her specific concern about the detrimental effect of whooping cough disease in the young population when stating, I have heard that whooping cough is very dangerous, especially for young children. I'm concerned that my child could get whooping cough and I wouldn't know what to do.

The next most common theme illuminated concerns about the high mortality rate associated with whooping cough. Participants clearly understood the danger of whooping cough as compared to other less deadly illnesses. One participant articulated his/her worry about whooping cough and the deadly effects on...children. Another participant exemplified this theme by stating [whooping cough is so]...serious...that it can lead to death.

In contrast to the first two themes, the final theme conveyed a lack of specific whooping cough concern. This group of participants acknowledged they knew little about whooping cough and, as a result, were unable to articulate a specific concern. As one participant stated, I honestly am not too sure. I don't know much about it. I just know it is bad. Another participant exemplified this theme with the statement, I don't really have a concern. I thought if [you're] unhealthy this is why [you] would need the shot but if you and your [family] are healthy [you] don't need it.

\section{New Knowledge}

As part of the posttest questionnaire, 93 participants shared what new knowledge they gained during the course of the education module. These participants offered 119 responses. The three most common themes were (1) an understanding of the seriousness of whooping cough $(n=30) ;(2)$ recognition of symptoms and treatment options $(n=30)$; and $(3)$ vaccination-specific information $(n=24)$.

Participants did not realize that whooping cough had such devastating effects. I didn't realize that $80 \%$ of children

Table 1. Pre- and Posttest Results on Likelihood to Vaccinate

\begin{tabular}{|c|c|c|c|c|c|}
\hline \multirow[b]{2}{*}{ Question } & \multirow[b]{2}{*}{$n$} & \multicolumn{2}{|c|}{ Pretest } & \multicolumn{2}{|c|}{ Posttest } \\
\hline & & $M$ & SD & $M$ & SD \\
\hline Vaccinate self & 98 & 78.3 & 32.1 & 91.5 & 21.5 \\
\hline Vaccinate child & 98 & 88.7 & 22.3 & 95.7 & 16.4 \\
\hline Vaccinate family & 98 & 65.0 & 37.8 & 84.1 & 28.7 \\
\hline
\end{tabular}




\section{Table 2. Comparison Between Pretest and Posttest Responses on Likelihood to Vaccinate}

\begin{tabular}{|c|c|c|c|c|c|c|c|c|}
\hline \multirow[b]{3}{*}{ Question } & \multicolumn{8}{|c|}{$95 \% \mathrm{CI}$} \\
\hline & Mean & & Mean & Diff & & & & \\
\hline & Diff & SD & LB* & $\mathbf{U B}^{* *}$ & $\boldsymbol{t}$ & df & $p$ & $d * * *$ \\
\hline Vaccinate self & -13.2 & 23.2 & -17.8 & -8.5 & -5.6 & 97 & .000 & .63 \\
\hline Vaccinate child & -6.9 & 21.8 & -11.3 & -2.5 & -3.1 & 97 & .002 & .33 \\
\hline Vaccinate family & -19.1 & 24.5 & -24.0 & -14.2 & -7.7 & 97 & .000 & .83 \\
\hline
\end{tabular}

* LB represents the lower bounds.

**UB represents the upper bounds.

***Cohen's $d$ was calculated using averaged standard deviations (Wiseheart, 2013).

under the age of 2 who contract whooping cough end up hospitalized to monitor breathing. Scary! As per another participant, I didn't realize it could be so serious for infants.

Thirty participants gained new knowledge in recognizing symptoms and treatment options for whooping cough. In the earliest stage of whooping cough, symptoms may be mistaken for a less severe illness; therefore, recognizing whooping cough symptoms seemed to resonate with these participants. [I learned] that whooping cough symptoms can start out as just a common cold. Participants also learned about the various treatment options once whooping cough was diagnosed. Whooping cough needs to be treated with antibiotics and cough medicine doesn't help.

The next most common theme was information specific to vaccinations. Knowledge gained by these participants centered on whooping cough vaccination rather than the disease. The important role of vaccination in preventing the spread of whooping cough was identified by participants, ...the only way to prevent [whooping cough] is by vaccination. Another participant expressed the importance of vaccinations by ensuring, ...everyone who comes in contact with the baby should be immunized.

\section{Remaining Questions}

As part of the posttest questionnaire, the final open-ended question asked participants to identify any whooping cough questions that remained after completing the education module. Participants $(n=36)$ offered 39 responses. There were two overarching themes that included specific questions about (1) the whooping cough vaccine $(n=20)$; and (2) the whooping cough disease $(n=16)$. The remaining responses were on miscellaneous topics $(n=3)$. The whooping cough vaccine theme was further categorized into five subthemes. These five subthemes included questions on vaccination (1) safety $(n=6) ;(2)$ schedule $(n=5)$; (3) education for families $(n=4)$; (4) cost $(n=3)$; and (5) availability $(n=2)$. One participant communicated the subtheme regarding vaccination safety when asking, What are possible symptoms from the vaccine? Another participant identified the subtheme regarding vaccination schedule by asking, Do non-pregnant adults need a whooping cough booster more than once in their adulthood? How long is the booster effective? Another participant embodied the subtheme of education for families by asking, How [do I educate] family members so they understand why I won't let them see the baby if they are not immunized?
The whooping cough disease theme was further categorized into three subthemes: (1) progression $(n=8)$; (2) mortality rate $(n=4)$; and (3) contagiousness $(n=4)$. One participant expressed the subtheme regarding disease progression by asking, Are there lifelong effects of having whooping cough? Another participant communicated the subtheme regarding mortality rate by asking, What is the infant mortality rate of those diagnosed with whooping cough? Another participant identified the subtheme of contagiousness by asking, How can I prevent my baby [from] getting whooping cough from strangers before they get vaccinated? Only three responses could not be categorized.

\section{Limitations}

There are limitations to our QI project. Although our data showed a significant increase in whooping cough knowledge with a whooping cough education module, the module was only evaluated among English-speaking WIC clients in one county in Utah. No demographic data were collected. Eligibility for WIC services is limited to families with a gross income at or below $185 \%$ of the Federal Poverty Level (USDA, 2016b); therefore, the module has not been evaluated among those with a higher income. Despite these limitations, we believe the module could be easily adapted for other populations.

We did not collect data about whooping cough vaccination rates among WIC clients before and after the module. While participants reported they were more likely to vaccinate themselves and their children, and recommend vaccination to other family members after reviewing the education module, we do not know if client behavior changed; namely, whether or not the participants followed through and vaccinated themselves or their children, or if family members were vaccinated. Long-term knowledge retention was not measured.

\section{Discussion}

The QI project on whooping cough education for WIC clients in the large Utah county was found to be effective at increasing immediate whooping cough knowledge. Given the success of the Utah WIC education module on increasing whooping cough knowledge, other WIC offices may also want to use the module. It could be an example for evaluating other WIC-related modules such as those educating parents on lead poisoning or hearing testing. When considering that the WIC program serves 9.7 million participants 


\section{Suggested Clinical Nursing Implications}

- The education module we developed on whooping cough can be adopted by maternal child nurses or nurse educators to provide patient education on whooping cough and the vaccine.

- The maternal child nurse can easily access the whooping cough education module online and can adapt as needed.

- Maternal child nurses may want to offer similar whooping cough information to parents because the parents in this project found the information helpful.

Parents indicated they planned to take action after completing the whooping cough education module by vaccinating themselves, their children, and their families.

per year (USDA, 2012) and 53\% of all infants born in the United States are served through the WIC program (USDA, 2015), there is great potential to provide accurate and effective vaccination education to millions of parents throughout the United States. If other WIC programs are interested in using the whooping cough education module, Whooping Cough, Serious for You...Deadly for Them, it is available at http://utahcounty.openwic.org/courses/immunizationsand-your-child-whooping-cough/whooping-cough/.

As part of the posttest questionnaire, participants were asked to identify any remaining whooping cough questions they had after completing the education module. Content analysis of these data revealed that participants still had a few remaining questions that were not adequately covered in the whooping cough module. Participants wanted additional information in a few areas such as cost of vaccine, vaccine safety concerns, and how to effectively provide whooping cough education for their family members. Although adding these topics to the existing education module may be helpful to some participants, such changes may have also made the education module more complex and longer in duration than the average WIC education module.

\section{Clinical Nursing Implications}

The target audience for this QI project was Utah WIC clients; however, the information in the whooping cough education module can be adopted by maternal child nurses or nurse educators to provide education to their patients on the whooping cough disease and vaccine. The module is easily accessible to maternal child nurses online. Many parents do not understand the devastating effects of whooping cough. Maternal child nurses are in a unique position where they can answer patients' questions on whooping cough thereby potentially influencing whooping cough vaccination rates. Maternal child nurses can use the WIC whooping cough education module as a resource when developing their own whooping cough education for expectant and new mothers. $\$$

\section{Acknowledgment}

The authors thank Brigham Young University Office of Research and Creative Activities.
Karlen E. Luthy is an Associate Professor, Brigham Young University, Provo, UT. The author can be reached viae-mailatbeth_lutby@byu.edu

Alicia Anderson is a Graduate Student, Brigham Young University, Provo, UT.

Janelle Macintosh is an Assistant Professor, Brigham Young University, Provo, UT.

Renea L. Beckstrand is a Professor, Brigham Young University, Provo, UT.

Lacey M. Eden is an Assistant Teaching Professor, Brigham Young University, Provo, UT.

Ryan Amy is an Operations Manager, Brigham Young University, Provo, UT.

Christopher I. Macintosh is a Clinical Modeling Engineer, Intermountain Healthcare, West Valley, UT.

The authors declare no conflicts of interest.

Supplemental digital content is available for this article. Direct URL citations appear in the printed text and are provided in the HTML and PDF versions of this article on the journal's Web site (www.monjournal.com).

Copyright (C) 2017 Wolters Kluwer Health, Inc. All rights reserved.

\section{DOI:10.1097/NMC.0000000000000358}

\section{References}

Centers for Disease Control and Prevention. (2014). Diphtheria, tetanus, and pertussis (DTaP) VIS. Retrieved from www.cdc.gov/vaccines/ hcp/vis/vis-statements/dtap.html

Centers for Disease Control and Prevention. (2016). Pertussis (whooping cough). Retrieved from https://www.cdc.gov/pertussis/

Centers for Disease Control and Prevention. (2017a). Pertussis (whooping cough) signs and symptoms. Retrieved from https://www.cdc. gov/pertussis/about/signs-symptoms.html

Centers for Disease Control and Prevention. (2017b). Help protect babies from whooping cough. Retrieved from https://www.cdc.gov/ features/pertussis/

Higley, C. (2013). \$2.5 million in emergency funding to reopen Utah WIC clinics. Retrieved from www.heraldextra.com/news/local/millionin-emergency-funding-to-reopen-utah-wic-clinics/article_0063f95a57be-5a54-9d36-ae386403b9c7.html

Leonard, W. (2012). Pertussis outbreak can be blamed on low vaccination rates: Utah experiencing highest incidence of whoop ing cough since 1946. Retrieved from www.deseretnews. com/article/865564282/Pertussis-outbreak-can-be-blamed-on-lowvaccination-rates.html?pg=all

Luthy, K. E., Miner, J. K., Faux, S. A., \& Sperhac, A. M. (2010). Improving immunization rates in the clinic and in the community. Contemporary Pediatrics, 27(9), 54-60.

United States Department of Agriculture. (2012). WIC participant and program characteristics 2012 final report. Retrieved from www.fns. usda.gov/sites/default/files/WICPC2012.pdf

United States Department of Agriculture. (2013). Women, infants and children (WIC). Retrieved from www.fns.usda.gov/wic/about-wicwics-mission

United States Department of Agriculture. (2015). Women, infants and children (WIC): About WIC - WIC at a glance. Retrieved from www. fns.usda.gov/wic/about-wic-wic-glance

United States Department of Agriculture. (2016a). The special supplemental nutrition program for women, infants and children (WIC program): Immunization screening and referral in WIC. Retrieved from https://www.fns.usda.gov/sites/default/files/wic/lmmunization $\% 20 F a c t \% 20$ Sheet.pdf

United States Department of Agriculture. (2016b). WIC income eligibility guidelines. Retrieved from www.fns.usda.gov/wic/wic-incomeeligibility-guidelines

Utah Department of Health. (2013). WIC 2012 participant satisfaction survey. Salt Lake City, UT: Author.

Wiseheart, M. (2013). Effectsize calculator. Retrieved fromwww.cognitive flexibility.org/effectsize/ 